\title{
lncRNAs - their potential in regulation of hypertension and behavior of ISIAH rats
}

\author{
Ivan Sidorenko \\ Laboratory of Molecular Plant \\ Pathology, \\ Institute of Cytology and Genetics, \\ Siberian Branch of Russian Academy \\ of Sciences, Novosibirsk, Russia \\ vanyasidorenko22@gmail.com
}

\author{
Vladimir Babenko \\ Laboratory of Human Molecular \\ Genetics, Institute of Cytology and \\ Genetics, Siberian Branch of Russian \\ Academy of Sciences, Novosibirsk, \\ Russia \\ bob@bionet.nsc.ru \\ Olga Redina \\ Laboratory. of Evolutional Genetics, \\ Institute of Cytology and Genetics, \\ Siberian Branch of Russian Academy \\ of Sciences, Novosibirsk, Russia \\ oredina@bionet.nsc.ru
}

\author{
Arcady Markel \\ Laboratory. of Evolutional Genetics, \\ Institute of Cytology and Genetics, \\ Siberian Branch of Russian Academy \\ of Sciences, Novosibirsk, Russia \\ markel@bionet.nsc.ru
}

\begin{abstract}
Long non-coding RNAs (IncRNAs) play an important role in the control of many biological processes in the body, including the development of cardiovascular diseases and hypertension. It is believed that IncRNAs play a central role in the epigenetic control of gene expression, however, the understanding of IncRNA biological functions and interactions is still far from being complete. In this work, we identified the IncRNAs differentially expressed in the hypothalami of hypertensive ISIAH and normotensive WAG rats, and revealed IncRNA-associated differentially expressed genes (DEGs) related to hypertension and behavioral characteristics of ISIAH rats (grooming, vertical activity, hyperactivity, abnormal emotion/affect behavior (including abnormal response to novelty). The work was carried out using transcriptome sequencing (RNA-Seq method). Three IncRNAs (Bc1, RGD1562890, and Snhg4) were found, the expression of which differed in the hypothalami of hypertensive ISIAH and normotensive WAG rats. The largest number of co-regulated genes, both associated with hypertension and behavior, was found for Snhg4. These findings may be useful for further understanding the role of IncRNAs in regulating the protein coding genes and modulating processes associated with both hypertension and behavior.
\end{abstract}

Keywords - RNA-Seq, IncRNA, hypertension, behavior, hypertensive ISIAH rats

\section{Introduction}

Long non-coding RNAs (lncRNAs) play an important role in the control of many biological processes in the body, including the development of cardiovascular diseases [1] and hypertension [2-5]. Although they have a central role in the epigenetic control of gene expression [4], the understanding of lncRNA biological functions and interactions is still far from being complete [5]. The development of NGS technologies, the results of which allow to identify the lncRNA-associated genes by calculating the correlation coefficient between lncRNA and mRNA expression levels [67], leads to a rapid increase in the number of studies in this field.

ISIAH rats model the stress-sensitive form of arterial hypertension. They are characterized by elevated blood pressure, characteristic structural and functional changes in target organs, increased stress sensitivity under the influence of mild emotional stress, and specific behavior in an unfamiliar environment. Increased activity of the hypothalamic-pituitary-adrenal and sympathetic adrenal systems suggest a key role for the hypothalamus in regulating the manifestation of the above phenotypic characteristics of hypertensive ISIAH rats [8]. Earlier, using transcriptome sequencing (RNA-Seq method), we determined genes differentially expressed in the hypothalami of hypertensive ISIAH and control normotensive WAG rats [9]. The current work presents the results of identification of differentially expressed lncRNAs in the hypothalami of the same rats and reveals the lncRNA-associated protein coding DEGs related to hypertension and behavioral phenotypes of ISIAH rats (grooming, vertical activity, hyperactivity, abnormal emotion/affect behavior and abnormal response to novelty).

\section{Methods}

\section{Animals}

The work was carried out using hypertensive ISIAH/Icgn (Inherited Stress Induced Arterial Hypertension) rats and normotensive WAG/GSto-Icgn (Wistar Albino Glaxo) rats at the age of 3 months. Rats were kept under standard conditions with free access to food and water. All animal experiments were approved by the Institute's Animal Care and Use Committee. Hypothalami from 3 rats in each group were collected and stored in RNA Later (Qiagen, Chatsworth, CA) at $-70 \mathrm{oC}$ until sequencing.

\section{RNA-Seq analysis}

The collected samples were sent to JSC Genoanalytica (Moscow, Russia), where transcriptome sequencing (RNASeq) was performed. The details of the protocol used were described earlier [9 4]. The data obtained were mapped to the RGSC Rnor_5.0 \rn5 reference genome. The Cufflinks / Cuffdiff programs were then used to reveal the differentially expressed transcripts. The $\mathrm{p}$ value cutoff for lncRNA was 0.01. The RNA-Seq data were deposited in the NCBI Short Read Archive database with Accession number PRJNA299102.

\section{Identification of the lncRNA-associated genes}

In order to obtain the lncRNA-mRNA co-expression pairs, the data (RPKM values) were log transformed, centered and normalized. Pearson correlation analysis was conducted between the lncRNAs expression and the expression profile of the protein-coding DEGs detected in the same samples. A 
correlation coefficient $>0.7$ was recognized as significant for the identification of the lncRNA-associated genes.

\section{Databases used}

Rat Genome Database (https://rgd.mcw.edu/) was used to identify the genes associated with the hypertension and behavioral phenotypes (behavior/neurological phenotype, grooming, vertical activity, hyperactivity, abnormal emotion/affect behavior (including abnormal response to novelty). Atlas of combinatorial transcriptional regulation in mouse and man [10] was used to reveal the DEGs encoding the transcription factor genes.

\section{Results}

Three lncRNAs (Bc1, RGD1562890, and Snhg4) were found, the expression of which differed in the hypothalami of hypertensive ISIAH and normotensive WAG rats (Table 1). The relationship between these IncRNAs and the expression level of protein coding DEGs associated with hypertension and behavioral phenotypes was calculated using the Pearson correlation coefficient. Among the hypothalamic protein coding DEGs that were associated with hypertension, one gene (Hyal1) associated with RGD1562890 $(\mathrm{r}=-0.73)$ and 12 DEGs associated with Snhg4 were found. Among them, the most significant association was found with the Igfbp2 gene ( $\mathrm{r}$ $=0.97)$, which is associated with insulin resistance in the development of hypertension.

Among the hypothalamic protein coding DEGs associated with behavioral phenotypes, one gene (Ercc2) associated with $\mathrm{Bc} 1(\mathrm{r}=0.88)$, two genes (Evc and Foxgl) associated with RGD1562890 ( $\mathrm{r}=-0.78$ and $\mathrm{r}=0.83)$, and 9 genes associated with Snhg4 were found. Among them, the most significant association was identified with the $I g f 2$ gene $(\mathrm{r}=0.88)$. It should be noted that the Ercc2 and Foxg1 genes encode transcription factors, and the $\operatorname{Ig} f 2$ gene is associated with cerebrovascular disorders.

TABLE 1 - The lncRNAs differentially expressed in the hypothalami of ISIAH and WAG rats

\begin{tabular}{|c|c|c|c|c|}
\hline \multirow[b]{2}{*}{$\operatorname{lncRNA}$} & \multicolumn{2}{|c|}{ Expression level in } & \multirow{2}{*}{$\begin{array}{c}\log 2 \\
\text { (fold_change) } \\
\text { ISIAH/WAG }\end{array}$} & \multirow[b]{2}{*}{ p_value } \\
\hline & $\begin{array}{l}\text { ISIAH, } \\
\text { RPKM }\end{array}$ & $\begin{array}{c}W A G, \\
R P K M\end{array}$ & & \\
\hline $\mathrm{Bc} 1$ & 137705.0 & 98177.6 & 0.49 & $1.85 \mathrm{E}-02$ \\
\hline RGD1562890 & 247.4 & 51.4 & 2.27 & $7.00 \mathrm{E}-04$ \\
\hline Snhg4 & 11.2 & 34.2 & -1.61 & $4.50 \mathrm{E}-03$ \\
\hline
\end{tabular}

\section{Conclusion}

In hypothalami of hypertensive ISIAH and control WAG rats, 3 differentially expressed lncRNAs and several lncRNAassociated protein-coding DEGs were found. The most highly expressed lncRNA ( $\mathrm{Bc} 1)$ is likely to influence the behavior of
ISIAH rats by regulating the expression of the Ercc2 gene, which encodes the transcription factor and is associated with abnormal emotion/affect behavior and abnormal response to novelty, but does not affect the regulation of genes directly associated with hypertension. According to our results, we can expect that lncRNA RGD1562890 is involved both in the regulation of processes associated with hypertension and in the regulation of behavior, in particular with hyperactivity, through the regulation of the Foxgl gene encoding the transcription factor. The largest number of co-regulated genes, both associated with hypertension and behavior, was found for Snhg4. These findings may be useful for further understanding the role of lncRNAs in regulating the protein coding genes and modulating processes associated with both hypertension and behavior.

\section{ACKNOWLEDGMENT}

The work was supported by Russian Foundation for Basic Research grant No. 20-04-00119a and by Budget project No. 0324-2019-0041-C-01.

\section{REFERENCES}

[1] S. Shen, H. Jiang, Y. Bei, et al., "Long Non-Coding RNAs in Cardiac Remodeling," Cell. Physiol. Biochem., vol. 41, no. 5, pp. 1830-1837, 2017.

[2] K. Gopalakrishnan, S. Kumarasamy, B. Mell, and B. Joe, "Genomewide identification of long noncoding RNAs in rat models of cardiovascular and renal disease," Hypertension, vol. 65, no. 1, pp. 200-210, 2015.

[3] L. Hou, Z. Lin, Y. Ni, et al., "Microarray expression profiling and gene ontology analysis of long non-coding RNAs in spontaneously hypertensive rats and their potential roles in the pathogenesis of hypertension," Mol. Med. Rep., vol. 13, no. 1, pp. 295-300, 2016.

[4] G. Wu, P.A. Jose, and C. Zeng, "Noncoding RNAs in the Regulatory Network of Hypertension," Hypertension, vol. 72, no. 5, pp. 10471059,2018

[5] C. Leimena and H. Qiu, "Non-Coding RNA in the Pathogenesis, Progression and Treatment of Hypertension," Int. J. Mol. Sci., vol. 19, no. 4, pii. E927, 2018.

[6] Y. Ma, T. Luo, D. Dong, et al., "Characterization of long non-coding RNAs to reveal potential prognostic biomarkers in hepatocellular carcinoma," Gene, vol. 663, pp. 148-156, 2018.

[7] L. Ma and C. Deng, "Identification of a novel four-lncRNA signature as a prognostic indicator in cirrhotic hepatocellular carcinoma," PeerJ, vol. 7, pp. e7413, 2019.

[8] A. L. Markel, O. E. Redina, M. A. Gilinsky, G. M. Dymshits, E. V. Kalashnikova, Y. V. Khvorostova, L. A. Fedoseeva, and G. S. Jacobson, "Neuroendocrine profiling in inherited stress-induced arterial hypertension rat strain with stress-sensitive arterial hypertension," J. Endocrinol., vol. 195, no. 3, pp. 439-450, 2007.

[9] L.O. Klimov, N.I. Ershov, V.M. Efimov, et al., "Genome-wide transcriptome analysis of hypothalamus in rats with inherited stressinduced arterial hypertension," BMC Genet., vol. 17, Suppl. 1., pp. 13, 2016.

[10] T. Ravasi, H. Suzuki, C.V. Cannistraci, et al., "An atlas of combinatorial transcriptional regulation in mouse and man," Cell, vol. 140, no. 5, pp. 744-752, 2010. 